\title{
ERRATUM
}

\section{Two streptothricins with a cis-streptolidine lactam moiety from Streptomyces sp. I08A 1776}

Maoluo Gan, Yan Guan, Xudong Zheng, Yanhui Yang, Xueqin Hao, Yishuang Liu, Liyan Yu and Chunling Xiao

The Journal of Antibiotics (2012) 65, 539; doi:10.1038/ja.2012.66

Correction to: The Journal of Antibiotics (2012) 65, 513-516; doi:10.1038/ja.2012.55

In the version of this article originally published online contained an error on the first page, Introduction section, fourth sentence. It has been corrected with the following:
But in recent years, some ST analogs with the carbamoyl located at C-12 were also isolated and identified from Streptomyces strains. ${ }^{6-9}$

The errors have now been rectified, and the correct article appears in this issue. The html and online pdf versions have also been rectified.

The publisher would like to apologize for the mistake. 